\title{
Research Staff Identifier
}

National Cancer Institute

\section{Source}

National Cancer Institute. Research Staff Identifier. NCI Thesaurus. Code C94063.

A unique symbol that establishes identity of the research staff. 